\title{
Interview
}

\section{Bildzensur und Recht. \\ Sabine Müller-Mall und Elisabeth Niekrenz im Gespräch mit Katja Müller-Helle}

Müller-Helle: Im November 2018 meldete Hyperallergic, dass der Facebook-Account des an der University of Texas lehrenden Kurators Ruben Cordova gelöscht wurde. ${ }^{1}$ Cordova hatte den Account über neun Jahre als fotografisches Archiv für seine kunsthistorischen Forschungen genutzt, um seine Bilder mit Annotationen und online verlinkten Vergleichsbeispielen in sozialen Netzwerken zu teilen und als Ressource für Vorträge in Museen und Universitäten zu nutzen. Der Facebook-Algorithmus hatte die fotografische Reproduktion von John De Andreas Self-Portrait with Sculpture von 1980, ein tradiertes kunsthistorisches Werk, als unangemessenen Inhalt erfasst und gelöscht. 入 Abb. 1 Die Skulptur erwies sich für die Mustererkennung als schwierig einzuordnendes Objekt: Die Oberflächenbehandlung der mit dem Material Polyvinyl und in Öl polychromierten Statue hatte eine solch hyperrealistische Darstellung eines weiblichen Körpers geschaffen, dass der Algorithmus nicht zwischen künstlerisch hergestelltem Artefakt und menschlichem Körper unterscheiden konnte. Hier scheint das Recht auf künstlerischen Ausdruck und die kuratorische Praxis von Cordova mit der normativen Löschungspraxis des Großkonzerns Facebook in Konflikt zu geraten. Aus bildwissenschaftlicher Sicht scheint mir vor allem problematisch, dass die in viele Richtungen weisende Polyvalenz von Bildern und Skulpturen, in diesem Fall die Verlebendigung der Skulptur durch eine spezifische Materialität und Oberflächenbehandlung, die ein mehrschichtiges Bedeutungsgefüge kreiert, durch den Algorithmus auf einen einzigen Aspekt reduziert wird: auf die Nacktheit. Frau Müller-Mall, wie verhält es sich hier aus verfassungstheoretischer Sicht mit der Kunst- und Meinungsfreiheit? Könnten Sie kurz skizzieren, wie im Fall Cordova, auch mit Blick auf die Differenz der Rechtssysteme in den USA und Deutschland, Normvorstellungen unter Druck geraten? Und wie sich hier die Mechanismen der Vereindeutigung, man könnte auch sagen: Bedeutungsverflachung, rechtstheoretisch einschätzen lassen?

Müller-Mall: Das ist ein schönes Beispiel, anhand dessen sich unterschiedliche Aspekte der Problematik besprechen lassen. Zunächst ist diese Verengung des Blickes auf bestimmte Aspekte, auf bestimmte Deutungsmöglichkeiten eines solchen Bildes, etwas, womit jede normative, jede bewertende Praxis zu tun hat, egal, ob das eine rechtliche Praxis ist oder eine andere. Recht verengt immer den Blick auf einen zu beurteilenden Gegenstand, wenn es bestimmte Aspekte für relevant erachtet und andere nicht. Ähnliches macht aber etwa auch eine ästhetische Beurteilung, die die 
Relevanzkriterien für Kunstwerke immer wieder neu bestimmt. Ganz allgemein gesagt, ist die Verengung der Perspektive ein gewöhnlicher Mechanismus, der mit der normativen Bewertung von Gegenständen, von Bildern, auch von Äußerungen, verbunden ist, der aber auch seinerseits beurteilt und kritisiert werden kann. In diesem Fall, wie auch im Falle von vielen anderen Sperrungen aufgrund von bestimmten Bildveröffentlichungen, erscheint die Verengung der Perspektive nun besonders drastisch. Ich glaube, bei diesen Fällen, bei denen es um die Darstellung von Nacktheit geht, kommen verschiedene Dinge zusammen. Das eine ist natürlich die Konzentration auf diesen Aspekt des Bildes - das ist sicher-

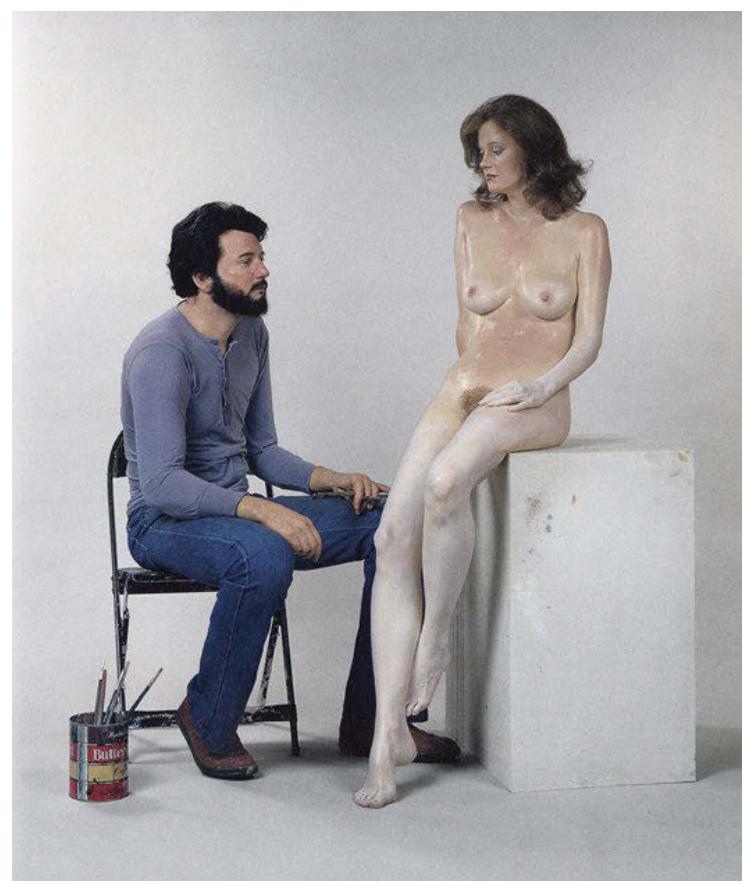

1: John De Andrea: Self-Portrait with Sculpture, 1980, Polyvinyl und Öl, 157,5×81,3 x 157,5 cm, Collection of Foster Goldstorm. lich das deutlichste. Das andere ist aber, dass natürlich verschiedene normative Grundierungen der verschiedenen Gesellschaften oder auch Rechtskulturen hier hineinspielen. Wir als Betrachter*innen, in diesem Fall geprägt in einer mitteleuropäischen oder sogar deutschen Rechtskultur, schauen anders auf den Fall als die amerikanische Durchschnittsgesellschaft, die bezüglich dessen, was man im amerikanischen Recht „obszön“ nennt, eine andere Schule durchlaufen hat. Verschiedene Bewertungsmechanismen prallen hier aufeinander und sie spiegeln sich auch in den rechtlichen Konstellationen oder in den rechtlichen Bewertungen.

Ganz grundsätzlich besteht bezüglich der Kunst- und Meinungsfreiheit wie bei jeder Art von rechtlicher Begrenzung von Äußerungen ein Problem darin, dass das Recht oder ein Gericht in einem konkreten Fall eine Äußerung oder ein Bild oder eine andere Art künstlerischer Darstellung auf bestimmte Aspekte reduziert, etwa solche, die mit Persönlichkeitsrechten in Konflikt geraten oder mit Urheber- oder Eigentumsrechten. Das können ganz unterschiedliche Konfliktgegenstände sein. Im Bereich der Kunst ist das Problem vielleicht noch größer als im Falle nicht-künstlerischer, sprachlicher Äußerungen, die von der Meinungsäußerungsfreiheit geschützt sind, weil wir typischerweise bei der Kunst eine größere Zahl von Aspekten haben, die relevant sein können, und eine gewis- 
se Deutungsoffenheit für die Gegenstände, um die es geht, die auch konstitutiv dafür ist, sie als Kunst zu beschreiben. Im Falle der Kunstfreiheit fallen dann nämlich nicht nur bestimmte Aspekte heraus, sondern es wird auch der Charakter des Gegenstandes, der als Kunst beurteilt wird, in Frage gestellt. Das heißt, in gewisser Weise ist das immer ein besonders drastischer Eingriff, weil er im Grunde den Gegenstand, Kunst als Kunst, selbst negiert oder unmöglich macht. Das ist eine andere Art von Problematik, als wir sie bei Meinungsäußerungen haben.

Ich würde aber in diesem besonderen Fall sagen, dass man sehr differenziert sehen muss, wie eigentlich das Unternehmen, der Konzern Facebook, mit dieser Situation umgeht. Und falls es einen Rechtsstreit gab, wie der ausgegangen ist. $^{2}$

Müller-Helle: Sie haben davon gesprochen, dass es verschiedene Rechtskulturen gibt, das amerikanische, deutsche oder auch das europäische Recht, denen verschiedene Normvorstellungen inhärent sind. Was mich interessiert ist die Tatsache, dass in diesem Fall nicht nur verschiedene Rechtssysteme in Konkurrenz geraten, sondern auch der technische Aspekt der automatischen Löschung im globalen Maßstab relevant wird, da Normvorstellungen jenseits juridischer Diskurse in die Filtersysteme und algorithmische Bilderkennung eingebaut sind. Deshalb würde ich gern überleiten zu Frau Niekrenz und zu einer netzpolitischen Perspektive. Für dieses Beispiel ist ja relevant, dass Pornografiefiltersysteme, CVPF-Verfahren (Computer Vision-based Pornography Filtering) Vorstellungen über Pornografie, Sexualität und den Körper eingeschrieben haben, die hier auf die klassische Kunstgeschichte und das Motiv Maler-Modell rückwirken. Die Normvorstellungen wirken durch die technischen Infrastrukturen hindurch und wirbeln die tradierten Kategorien durcheinander. Deswegen würde ich gerne anhand dieses Beispiels fragen, was Sie zum technischen Aspekt, der technischen Einhegung von dargestellter Nacktheit sagen?

Niekrenz: Ich denke, an diesem Beispiel zeigen sich zwei der Kernprobleme, die entstehen, wenn Bilder oder andere Inhalte automatisiert erfasst und in die Kategorien „erlaubt“ und „nicht erlaubt“eingeteilt werden: Auf der einen Seite geschehen schlicht Fehler. Sie hatten dargelegt, dass Facebooks Algorithmen in der Fotografie das Abbild einer realen nackten Frau erkannt haben, obwohl es sich tatsächlich um die Fotografie einer Plastik handelte. Da stellt sich die Frage, ob Facebooks Nutzungsbedingungen nur eine Nacktheitsdarstellung, die die Fotografie eines realen Menschen ist, verbieten oder auch Statuen.

Müller-Helle: Ich kann kurz aus den Facebook-Richtlinien zitieren. Es geht genau um diese Unterscheidung in Facebooks Community Standards, dort wird explizit 
gesagt: „We also allow photographs of paintings, sculptures and other art that depicts nude figures. ${ }^{\text {3 }}$

Niekrenz: Also handelt es sich um einen schlichten Fehler des technischen Systems. Da ließe sich vielleicht noch argumentieren, dass sich die Technik in den nächsten Jahren verbessern könnte. Ein anderes Problem, das sich hier zeigt und das meiner Ansicht nach auch nicht mit neuen Entwicklungen lösbar ist, ist das der Güterabwägung, die der Algorithmus nicht vornehmen kann. Algorithmen können darauf trainiert werden, bestimmte Dinge in Abbildungen zu erkennen. Etwa durch Machine Learning, bei dem Algorithmen nicht regelbasiert arbeiten, sondern anhand einer Vielzahl von Trainingsdaten ,lernen': Man speist zum Beispiel eine Vielzahl von Bildern von Brustwarzen und von Bildern, die keine Brustwarzen darstellen, ein. So kann der Algorithmus selbst Kriterien finden, die zum Teil auch die Entwickler*in nicht nachvollziehen kann. So kann ein technisches System mit einer gewissen Zuverlässigkeit eine Brustwarze, eine IS-Flagge, einen Hund oder eine Katze erkennen. Was diese Technologie nicht leisten kann, das ist die juristisch gebotene Kontextualisierung und Güterabwägung. Nachdem Nacktheit erkannt wurde, muss schließlich der gesamte soziale Kontext, in diesem Fall, dass es sich um ein Kunstwerk handelt, einbezogen werden. Das spielt für Kunst eine große Rolle, aber insbesondere auch für politische Äußerungen. Diese Nacktheit, die hier erkannt worden ist, hat eine Berechtigung, gezeigt zu werden. Die Einbeziehung des Kontextes und die Güterabwägung sind Praxen, die so etwas wie ein soziales Verstehen erfordern und die algorithmisch nicht geleistet werden können.

Müller-Helle: Da möchte ich einhaken und bemerken, dass Facebook, Google oder Twitter zwar technisch löschen, gleichzeitig aber menschliche Abwägung, also soziale Kontextualisierung einführen, indem sie automatische Löschmechanismen mit Content Moderation kombinieren. Das Problem ist aber, dass die Moderator*innen selber normative Voreinstellungen gemäß den Unternehmensrichtlinien umsetzen. Diese Praxis der „Reinigung des Netzes“ steht der Rhetorik von Netzneutralität entgegen, also einer von den Großkonzernen behaupteten Neutralität in der Bereitstellung sozialer Kontaktmöglichkeiten, was Auswirkungen auf die moralische, ethische und politische Wirksamkeit der Bilder hat. Diesbezüglich würde ich gerne ein Zitat in die Runde werfen, das auf die Frage zielt, was diese technisch implementierten, aber nicht öffentlich diskutierten Regularien eigentlich für Kulturschaffende bedeuten. Hanno Rauterberg hat in seinem Buch Wie frei ist die Kunst? Der neue Kulturkampf und die Krise des Liberalismus von 2018 vermutet, dass es durch diese Form der Regulation in der Digitalmoderne zu einer Konsenskultur kommen wird und deswegen Kulturschaffende ihre Praxis verändern. Es gibt Kurator*innen, die ihre streitbare 
Kunst abhängen, also Selbstzensur betreiben, weil sie Angst haben, dass diese sie ihren Job kosten könnte. Würden Sie die Einschätzung teilen, dass die neuen Zensurmechanismen eine Einheitskunst oder Konsenskultur vorantreiben?

Niekrenz: Ich bin mir tatsächlich nicht sicher, ob man das so feststellen kann. Es finden sich online mannigfaltige kreative Formen, die sich manchmal auch anhand von Zensurpraktiken weiterentwickeln. Ich will das nicht verherrlichen. Aber nehmen wir das Beispiel TikTok, eine chinesische App, auf der vor allem kurze Videos verbreitet werden. Nach Recherchen von netzpolitik.org werden dort Inhalte weniger gesperrt oder völlig gelöscht, als vielmehr in ihrer Reichweite gedrosselt , wenn sie bestimmte politisch oder aus anderweitigen Gründen nicht erwünschte Inhalte enthalten. Das heißt, es wird dafür gesorgt, dass diese Beiträge nicht in sehr vielen Newsfeeds von Personen erscheinen. Vielleicht ist das eine noch hinterhältigere Form von Zensur, als die, bei der das Bild überhaupt nicht mehr sichtbar ist, weil auch derjenige, der es hochgeladen hat, nicht mitbekommt, dass etwas gedrosselt wurde. Auf TikTok ging 2019 ein Video viral, das auf der Bildebene die Nutzer*in bei der Verwendung einer Wimpernzange zeigte getarnt als Beauty-Tutorial sprach sie in dem Clip aber über die Unterdrückung von Uiguren in China. Im Allgemeinen werden auf Social Media gegenwärtig Inhalte in großem Umfang hochgeladen, auch auf die Gefahr hin, dass etwas gelöscht wird. Ich kann mir vorstellen, dass sich das ändern wird, wenn die europäische Urheberrechtsrichtlinie umgesetzt wird.

Müller-Mall: Ja, das ist nochmal eine ganz eigene Frage, die Sie gerade angesprochen haben, was es für sogenannte Chilling Effects gibt. Ich würde gerne auf Ihre Ausgangsfrage zurückkommen, Frau Müller-Helle. Ich finde, es sind unterschiedliche Punkte, die darin auftauchen. Das eine ist der Begriff der Konsenskultur. Ich habe mit dem Begriff relativ große Schwierigkeiten, ähnlich große Schwierigkeiten wie mit dem der Cancel Culture. Die mit diesem Begriff beschriebene Entwicklung lässt sich in meinen Augen empirisch überhaupt nicht belegen. Es gibt zwar das Phänomen, dass Ausstellungen sogar im Extremfall abgesagt werden oder dass Kontextualisierungen zu Bildern in die Kuratierung von Ausstellungen hineingeraten, die sonst nicht vorgesehen waren, und auch, dass die Angst vor Protesten bestimmte Diskussionen hervorruft. Gleichzeitig können wir aber auf sozialen Netzwerken ganz gut beobachten, dass es einen Streit um Kunst gibt, was für sich genommen nichts Neues ist. Das ist aus meiner Sicht kein besonderes Phänomen dieser Zeit, sondern man hat das, wenn man etwa in die 1960er- oder die 1970er-Jahre schaut, früher genauso gehabt oder mit den Diskussionen um Pornografie in den 1980er-Jahren in den USA, der Frage danach, inwieweit Pornografie Kunst sein kann oder eben Gewalt oder Gewaltandrohung 
ist. Solche Arten von sehr grundsätzlichen Diskussionen um Kunst entlang spezieller Kunstwerke gibt es natürlich immer wieder. Ich würde eher sagen, wir leben in einer Zeit, in der wir diesen Streit um Kunst gut beobachten können. Ich würde deswegen diesen Begriff der Konsenskultur erst einmal zurückweisen. Natürlich kann die Angst vor streitbarer Kunst, die Rauterberg nennt, trotzdem relevant sein. Es ist aber immer die Frage, wie sie diskutiert wird, wie zum Beispiel im Fall des Künstlers Balthus und seiner Thérèse rêvant. Da ging es vor allem um das Nichtzeigen, etwa in Basel, als Balthus in der Fondation Beyerle ausgestellt wurde, auch darum, Kontextualisierungen einzuziehen, bestimmte Perspektiven in der Rezeption zu diskutieren. Das würde ich erst einmal alles unter das Phänomen ,Streit um Kunst' einordnen und nicht unter dem Stichwort Konsenskultur. Was ganz bestimmt eine Rolle spielt, auch bei der Dynamik eskalativer Strukturen, die solche Diskussionen um Kunst haben, sind die technischen Bedingungen und auch die Möglichkeiten von sozialen Medien, die eine andere Art von Öffentlichkeitsstruktur hervorbringen. Es ist vielleicht ein Allgemeinplatz, dass wir mit sehr vielen verschiedenen Vorstellungen von Öffentlichkeit denken können und auch denken müssen, dass es beispielsweise diese Unvorhersehbarkeit in der Frage gibt, wann plötzlich eine Frage oder eine Kritik oder eine Äußerung oder eben ein Bild eine besondere Öffentlichkeit bekommt und sich dann ein Streit darum entwickelt und wann das vielleicht auch nicht passiert. Ich glaube, da haben wir es sozusagen mit einer Art von Unvorhersehbarkeit oder auch von Potenzialität zu tun, die in gewisser Weise neu ist und die ganz stark mit der Verbreitungslogik von digitalen Kommunikationsstrukturen zu tun hat.

$\mathrm{Zu}$ einem anderen von Ihnen angesprochenen Aspekt, zur Netzneutralität, würde ich der Vollständigkeit halber noch gerne etwas anfügen, ohne dass es eigentlich meine Position wäre. Man muss sich klarmachen, dass Facebook ja kein die Öffentlichkeit strukturierender Staat ist, sondern ein privater Konzern mit unglaublicher Marktmacht. Es geht um konsumatorische Angebote. Man kann hier zwar durchaus ganz ernsthaft darüber nachdenken, ob man diese Art von Konzernen wirklich vollständig abgrenzen kann von öffentlichen Strukturen. Aber man muss trotzdem auch anmerken, dass hier ein privater Konzern ein konsumatorisches Angebot macht; es ist erst einmal auch meine Freiheit, ob ich dieses Angebot nutze oder mich davon fernhalte. Das ist kein gutes Argument, darüber könnten wir auch gleich noch diskutieren, aber ich glaube, dass es wichtig ist, es im ersten Schritt zu nennen, um auch nochmal einen zweiten Schritt offen zu legen. Es geht ja nicht nur um diese Art von nachgängigen Löschungs- oder Sperrungsentscheidungen. Die Art und Weise, wie wir kommunizieren, wie wir uns auch sozial verstehen und wie wir vielleicht dann auch - unbewertet gesagt - bestimmte kulturelle Wertungen weiterentwickeln, wird generell von den Angeboten solcher Konzerne mitgeprägt: Nicht nur nachgängige Löschungsentscheidungen spielen 
hier eine Rolle, sondern all das, was man Affordanzen nennt. Was mir das Design, die Struktur dieser Netzwerke anbietet, strukturiert ja auch meine Kommunikation und meine Art und Weise, wie ich bestimmte soziale Strukturen, Momente, Begegnungen denke oder lerne. Das heißt, wir haben auf ganz vielen Ebenen Eingriffe in soziale Kommunikation, und für mich wäre die eigentliche Frage, ob wir diese Eingriffe unterschiedlich behandeln müssen, und wenn ja, an welchen Stellen: nachgängig oder vorgängig, privat oder öffentlich, generell oder jeweilig.

Müller-Helle: Was auch die Unterscheidung von Vor- und Nachzensur aufruft, die im Diskurs zu Zensurpraktiken sehr entscheidend ist. Es ist ein bekanntes Argument zu sagen, dass ich mich verweigern kann, soziale Netzwerke wie Facebook oder Twitter überhaupt zu nutzen. Ich habe Kolleg*innen, die das entschieden so machen, die sagen: „Nein, ich werde mich nicht hineinbegeben in diese technische Sozialität.“ Aber ich finde, das trifft zusammen mit einer anderen Größe, die von Frau Niekrenz in ihrem Papier zu Uploadfiltern und Netzkultur beschrieben wird, nämlich, dass die Gefahr schon besteht, dass es kein Außen mehr gibt von technischen Infrastrukturen. ${ }^{4}$ Sie haben hierfür den Begriff der Zensurinfrastruktur eingeführt. Unsere gesamte Sozialisierung und unsere Art des sozialen Austauschs, die Kommunikation ist durchdrungen von technischen Umgebungen, deren Operationalität selbst opak bleibt. Und das ist natürlich gar nicht anders möglich. Angesichts eines Bilddatenvolumens von 1,4 Billionen Bildern, die 2020 wahrscheinlich gemacht und potenziell über soziale Netzwerke geteilt werden, muss es ja irgendeine Art von Regulierung geben. Weil natürlich, wie Sie es eingangs auch schon gesagt haben, auf Jugendschutz geachtet und Persönlichkeitsrechte gewahrt werden müssen. Gleichzeitig ist jetzt die Frage, wie wir mit dem Moment der von Frau Müller-Mall eingeführten ,freien`Entscheidung auch gegen solche sozialen Netzwerke umgehen.

Niekrenz: Den Hintergrund zum Begriff der Zensurinfrastruktur kann man ganz gut beschreiben anhand der Regulierung von als terroristisch eingeordneten Inhalten. Als Anfang der 2010er-Jahre der sogenannte ,Islamische Staat' immer stärker wurde, wurde auch seine Online-Propaganda immer stärker sichtbar. Damals konnte man beispielsweise Enthauptungsvideos auf YouTube finden. Es kam ein politischer Prozess in Bewegung, bei dem die USA und die EU-Kommission die großen Netzwerke aufgefordert haben, dagegen aktiv zu werden. So fand eine Art staatlich angeregte Selbstregulierung statt, die kürzlich in der Gründung des GIFCT (Global Internet Forum to Counter Terrorism) institutionalisiert wurde. Das ist ein Zusammenschluss von großen Netzwerken, mittlerweile gehören um die zwanzig dazu, die in den letzten Jahren eine gemeinsame Infrastruktur eingeführt haben, um terroristische Inhalte von ihren Plattformen fernzuhalten, 
was, im Zuge des Christchurch-Attentats 2019 und weiterer Attentate, die live gestreamt worden sind, starken Auftrieb erfahren hat. ${ }^{5}$ Diese Institution betreibt eine Datenbank mit Hashwerten, sogenannten Fingerprints, von Inhalten, insbesondere von Videos und Fotografien, die als Terrordarstellungen klassifiziert worden sind. Bei diesen Hashwerten handelt es sich um so etwas wie Prüfsummen von markanten Merkmalen von Audio- und Videospuren oder Bildern. Anhand dieser Werte können Inhalte wiedererkannt werden, auch wenn die entsprechenden Dateien ein wenig verändert sind, zum Beispiel durch Farbfilter oder Rauschen. Es können also nicht nur bitweise identische Dateien wieder erkannt werden. Lädt eine User*in einen Inhalt hoch, der einmal als terroristisch klassifiziert und in diese Datenbank eingefügt wurde, wird dieser Inhalt nicht zugelassen. Es ist schwer, sich mit rechtlichen Mitteln dagegen zu wehren. Dabei kommt es zu verschiedenen Problemen: Die NGO The Syrian Archive zum Beispiel dokumentiert Kriegsverbrechen im syrischen Bürgerkrieg und hat im Zuge dessen Tausende Videos auf YouTube hochgeladen, die dazu beitragen sollten, Täter*innen verantwortlich zu machen. Youtube hat 2017 eine Vielzahl dieser Videos gelöscht. Ausgehend von einem Unterfangen, das im Kern respektabel ist, wurde eine technische Infrastruktur geschaffen, die keinerlei demokratischer oder gerichtlicher Kontrolle unterliegt, fehleranfällig und vollkommen intransparent ist. Diese Infrastruktur unterliegt derzeit sehr verschiedenen Machteinwirkungen von Unternehmen, sehr unterschiedlichen Staaten und Staatenverbunden. Aber wozu könnten autoritäre Staaten diese Infrastruktur verwenden, wenn sie ihre Macht über sie ausdehnen könnten?

Müller-Helle: Sie beschreiben, dass es eine Gemengelage ist, also verschiedenste Institutionen und Machtinteressen in den Zensurinfrastrukturen wirksam sind. Frau Müller-Mall hatte vorhin eine Historisierung angedeutet und beschrieben, dass es historisch immer schon Institutionen der Zensur gab. Könnte man diese beiden Aspekte zusammenführen? Kann man die Frage nach der aktuellen technischen Zensur nicht in historischer Perspektive noch mal neu auffächern? Zum Beispiel musste auch Goya seine nackte Maja der Spanischen Inquisition vorlegen, was natürlich eine extrem wirksame, sehr mächtige Institution der Zensur war. Dann gibt es Beispiele von zirkulierenden Feldpostkarten im Ersten Weltkrieg Hunger-, Schmäh- und Karikaturpostkarten, die von eigens zu diesem Zweck eingerichteten Zensurbehörden in München und Berlin einbehalten wurden, um der offiziellen Kaiserpropaganda nicht zu schaden. Ein drittes Beispiel, mit dem Sie sich auch beschäftigt haben, Frau Müller-Mall, sind die Strauß-Karikaturen von 1980/81, als der damalige bayerische Ministerpräsident Franz Josef Strauß vor dem Bundesverfassungsgericht klagte, weil der Karikaturist Rainer Hachfeld ihn als kopulierendes Schwein dargestellt hatte. Dies sind drei historisch sehr 
verschiedene, aber markante Beispiele, an denen man sehen kann, dass diese Aufregung, in der wir uns heute hinsichtlich der Inhaltsregulierungen oder Filterungen befinden, historisch unterfüttert werden müsste. Sie haben ja differenziert beschrieben, dass in historischer Perspektive immer starke Zensurinstanzen wirksam waren, die das, was gezeigt werden kann, und das, was gesagt werden darf, gerade in den Feldern der Karikatur, der Kunst und des Politischen, geregelt haben. Wie unterscheidet sich die heutige Lage von historischen Beispielen?

Müller-Mall: Die Fälle, die wir vorher besprochen haben und die, die Sie jetzt genannt haben, unterscheiden sich in ganz vielen Punkten. Das Zentrale ist aus meiner Sicht, dass teilweise Staaten oder eine politische Gewalt beteiligt sind und teilweise private Konzerne Löschungen oder Sperrungen vornehmen. Rein rechtsbegrifflich würde man im engeren Sinne nur das staatliche Handeln als Zensur beschreiben, das private Handeln erst einmal nicht. Wobei man dann genauer hingucken müsste, wie das private Handeln staatlich indiziert ist, was es für eine Art von Zusammenspiel gibt oder welche Wirkmacht es hat. Diese Arten von Zensur, die Sie jetzt beschrieben haben, sind ganz unterschiedlich, aber jeweils eben auch nochmal zu unterscheiden von dem, was Social-Media-Konzerne tun, weil es etwas anderes ist, ob mir durch eine öffentliche Gewalt verboten ist, ein bestimmtes Bild zu drucken, als Medium beispielsweise, oder ob Facebook mir nicht erlaubt, dasselbe Bild zu verbreiten, ich es aber genauso gut auf andere Weise verbreiten kann. Und es hat natürlich auch politisch eine andere Auswirkung, wenn die öffentliche Gewalt, der ich in gewisser Weise vertrauen muss oder ausgeliefert bin, auf meine Äußerungsfreiheiten zugreift. Der Unterschied wäre mir schon wichtig. Dahingehend ist zu betonen, dass wir uns in den meisten westlichen Staaten im Moment sicherlich nach wie vor in Verfassungssystemen befinden, die eine staatliche Vorzensur ausschließen. Das ist im Grundgesetz und in vielen anderen Verfassungen ganz explizit geregelt. Das heißt natürlich nicht, dass eine Nachzensur, also die gerichtliche Aufhebung von bestimmten Erlaubnissen, dann grundsätzlich ausgeschlossen ist, sondern es gibt genau das, was Frau Niekrenz eine Güterabwägung genannt hat, durchaus als das Recht des Rechts, wenn nämlich bestimmte gleichrangige Rechte gegeneinander stehen, etwa die Äußerungsfreiheit der einen und das Persönlichkeitsrecht des anderen. Dies muss dann in eine Abwägung treten, wobei auch die Äußerungsfreiheit den Kürzeren ziehen und dann rechtlich gesehen sowas wie eine Nachzensur vollzogen werden kann. Die historischen Beispiele zeigen diesbezüglich unterschiedliche Konstellationen. Die Inquisition hat nochmal eine andere Intransparenz oder Unzugänglichkeit als eine gerichtliche Entscheidung in Deutschland in den 1980er-Jahren, die in der Regel anfechtbar ist. Das bundesverfassungsgerichtliche Urteil im Fall der Strauß-Karikaturen war nur das letzte Urteil in einer Reihe 
von Urteilen, und die Sache ist auch grundsätzlich von Fall zu Fall immer wieder neu zu bewerten. Das heißt nicht, dass seit diesem Urteil tierische Darstellungen von Politikern untersagt wären oder Karikaturen von Politikern untersagt wären. Und es ist ein ganz anderer Fall als die politische Steuerung von Berichterstattungen, wie etwa im Zusammenhang mit dem Ersten Weltkrieg. Dort ging es nicht nur darum, einzelne Brief- oder Postkartenkontakte zu unterbinden, sondern auch darum, Informationen über die Lage des Krieges vorzuenthalten, und zwar einer großen Gruppe vorzuenthalten. Die verschiedenen Fälle haben aber natürlich letztlich gemein, dass sie entweder die Grenzen von Äußerungsfreiheiten verhandeln oder eine Verhandlung dieser Grenzen nicht mehr zulassen.

Müller-Helle: Ich würde darüber hinaus sagen, dass sich alle diese Beispiele, die ich exemplarisch aus den historischen Kontexten herausgelöst habe, von den technischen Zensurmaßnahmen heute vor allem darin unterscheiden, dass sie überhaupt in öffentlichen Debatten verhandelt wurden oder eine Zurschaustellung von den Instrumenten der Zensur stattfand, wie im Fall der Spanischen Inquisition, also die Kriterien zur Beurteilung öffentlich sichtbar wurden. Wenn man es von der Debatte um die Sichtbarkeit bestimmter Zensurmechanismen her sieht, gibt es eine neue Form der Opazität und eine Verunklärung dessen, was wo gelöscht wurde. Dass mir noch nicht einmal vorgeführt wird, wie im Facebook-Fall von John de Andrea, dass hier zensiert wird, finde ich eine neue Qualität von Regulierung. Auch durch DeepfakeVerfahren kommen bestimmte Löschungen gar nicht mehr vor, die zuvor durch Zensurmarker wie Balken oder Verpixelung als zensiert gekennzeichnet worden waren; sie gelangen einfach nicht mehr ins Feld der Sichtbarkeit. Das ist ja ein Phänomen, welches anhand des Dokumentarfilms The Cleaners. Im Schatten der Netzwelt (2018) von Hans Block und Moritz Riesewieck diskutiert wurde. Die Arbeiter*innen in Manila, die arbeitsrechtlich in einem ganz schwierigen Verhältnis zu den US-Großkonzernen stehen, schauen 25.000 Bilder am Tag an, um Bilder zu löschen, die dann im ,gereinigten' Newsfeed gar nicht mehr erscheinen. Bezüglich der Sichtbarkeit der Löschungsvorgänge ist eine neue Praxis gegeben, die man anders fassen müsste als eine Debatte um die Grenzen des Zeigbaren, wie sie vor Gericht geführt werden kann. Noch ein weiterer Punkt: Sie sagen, was natürlich sehr wichtig ist, dass die staatliche Beteiligung, also staatliche Zensur, etwas völlig anderes ist als die privater Konzerne, weil diese Richtlinien nach eigenem Ermessen etablieren können. Gleichzeitig gibt es meines Erachtens eine Umverteilung der Machtstruktur, in dem Sinne, dass die Content Moderation der genannten Großkonzerne gegenüber den staatlichen Regulierungen in der Digitalmoderne an Bedeutung gewonnen haben. 
Müller-Mall: Ganz grundsätzlich haben wir, das sehe ich ganz genauso, gegenwärtig andere Möglichkeiten, was die Regime von Sichtbarkeit oder Sichtbarmachung und das Teilen und Verbreiten angeht, als in anderen Gesellschaften und zu anderen Zeiten. Ich würde aber auch sagen, dass die Opazität von solchen Entscheidungen, die Sie beschreiben, gerade ein typisches Merkmal von Zensur ist, und zwar auch von staatlicher Zensur, und es auch historisch immer war. Es ist dann doch, wenn wir uns eine staatliche Zensur in einem autoritären oder diktatorischen oder faschistischen System in der ersten Hälfte des 20. Jahrhunderts vorstellen, noch viel stärker ausgeprägt als etwa bei Twitter, wo ich immer noch bestimmte Sperrungen sehen kann, auch wenn ich an vielen Stellen keine Kriteriologie erstellen und nicht abbilden kann, was tatsächlich gelöscht wird und was nicht. Umgekehrt ist es gerade und immer schon das Wesen von staatlicher Zensur, dass sie Sichtbarkeit in einer Weise unmöglich macht. Die staatliche Zensur, beispielweise in einem autoritären System, ist typischerweise so ausgeprägt, dass sie nicht erst geplante Ausstellungen verhindert, sondern vorher einsetzt. Die Zensur geplanter oder schon laufender Ausstellungen gibt es natürlich auch in jüngerer Zeit, wenn wir zum Bespiel an das Russland der Nullerjahre, etwa die Ausstellung Vorsicht, Religion! denken. Die Zensursituation ist aber typischerweise davon geprägt, dass die Idee zu einer bestimmten Ausstellung überhaupt nicht an die Öffentlichkeit gelangen kann, weil sie so früh im Keim erstickt wird durch die Zensur oder es unmöglich gemacht wird, ein bestimmtes Bild überhaupt anzufertigen. Viele Eingriffe finden so früh statt, dass sie am Ende überhaupt nicht mehr sichtbar werden, aber trotzdem eine zensorische Wirkung haben. Anders gesagt: Die Unsichtbarkeit dieser Prozesse ist ein Teil von Zensur. Die Frage ist eher, was wir an dem Umgang von SocialMedia-Großkonzernen mit Bildern oder mit Äußerungen darin wiedererkennen können und ob dies vergleichbar ist. Oder ist es hier vielmehr, wie Sie angedeutet haben, den technischen Bedingungen geschuldet? Denn natürlich, das sehe ich auch so, reichen die algorithmischen Möglichkeiten, auch bei allen algorithmischen Lernmethoden in den letzten Jahren, lange nicht aus, um angemessen zu differenzieren. Ich würde auch sagen, ganz ähnlich wie Frau Niekrenz zu Beginn unseres Gesprächs, dass es ab einem gewissen Punkt sowohl bei Bildern als auch bei Äußerungen der Fähigkeit des Urteilens bedarf. Wir müssen die Bilder beurteilen, um aus den diversen Aspekten, die sie möglicherweise sichtbar machen, diejenigen herauszufiltern, mit denen wir sie dann schließlich bewerten oder einordnen. Diese Möglichkeit zur Beurteilung haben Algorithmen eben nicht. Sie können Mustererkennung betreiben, sie können Kriterien finden, um zu unterscheiden, aber sie müssen eben spezifische Kriterien zur Klassifikation oder zur Differenzierung finden. Wir können dagegen urteilen, ohne dass wir das Allgemeine, unter dem wir beurteilen, bestimmen können. Das ist eine ganz andere Fähigkeit, die wir Menschen haben, um mit Gegenständen umzugehen. Die wird man über ein 
statistisches Verfahren, was die algorithmische Kriterienbildung letztlich ist, nie erreichen können. In dieser Hinsicht teile ich Ihre Kritik oder Skepsis.

Müller-Helle: Dann ist, wenn man Ihren Ausführungen folgt, Opazität eigentlich der Normalfall von Zensur. Das hieße wiederum, dass die Rede von der Sichtbarkeit der Bilder, der Bilderflut oder der immer und überall versendbaren Bilder zu relativieren ist. Der theoretischen Figur, die Vilém Flusser im Ins Universum der technischen Bilder (1984) prominent beschrieben hat, dass alle Bilder elektronisch immer und überall hin versendet werden können, müsste dann diese andere, für uns im Gespräch relevante Figur der Opazität und der Löschung beigestellt werden.

Niekrenz: Das Neue an den aktuellen Zensurpraktiken ist meines Erachtens nicht ihre Opazität, sondern die zugrunde liegende Reichweite des Individuums, auch des status- und vermögenslosen Individuums, und seine Möglichkeit, Bilder zu verbreiten, die das Internet historisch erstmalig eröffnet hat. Diese Reichweite hat zunächst für politisches Empowerment gesorgt, gerade in problematischen Regimen. Dies ist ein Aspekt des Internets, den wir erhalten sollten. Zumindest sollte diese Reichweite, diese Sendefähigkeit nur auf möglichst demokratische, möglichst transparente Art und Weise eingeschränkt werden.

Müller-Helle: Was würden Sie konkret sagen, welche Effekte der Artikel 17 der Urheberrechtsreform auf diese Möglichkeiten des demokratischen Gebrauchs des Internets hat?

Niekrenz: Tatsächlich sehr einschränkende. Eine Möglichkeit des demokratischen Gebrauchs des Internets ist ja vor allem durch Internetplattformen geschaffen worden, also dadurch, dass es Anbieter gibt, die Webseiten programmieren und Serverplatz bereitstellen, die alle Personen nutzen können, um mit sehr wenig technischen Voraussetzungen Inhalte zu publizieren. Dieses Plattformmodell ist zumindest in der Europäischen Union ganz wesentlich durch die E-CommerceRichtlinie von 2001 geprägt worden und durch das darin niedergelegte sogenannte Provider-Privileg. Es besagt, dass Online-Plattformen grundsätzlich nicht haften für Beiträge, die auf ihnen gepostet wurden, solange sie nicht darauf hingewiesen wurden, dass ein Inhalt aus irgendwelchen Gründen illegal ist oder Rechte anderer beeinträchtigt („,notice and takedown“). Artikel 17 der Urheberrechtsreform bricht mit diesem Paradigma. Die Norm besagt unter anderem, dass Plattformen gewährleisten müssen, dass ein Inhalt nicht erscheint, wenn die Rechteinhaber*in der Plattform Erkennungsinformationen über diesen Inhalt gesendet hat. Das heißt, die Plattform muss jeden neuen Inhalt, bevor er hochgeladen wird, daraufhin überprüfen, ob er mit diesem Inhalt übereinstimmt. Das ist eine kom- 
plette Abkehr von dem Gedanken, dass man erst einmal alles stehen lassen darf. Sie macht die Implementierung eines sogenannten Uploadfilters notwendig. Das Problem ist aber nicht nur die Urheberrechtsreform. Der EU-Gesetzgeber arbeitet an einer Verordnung, die terroristische Inhalte aus dem Netz verbannen will und ebenfalls Uploadfilter verpflichtend machen könnte. In diesem Fall werden sich möglicherweise sehr viele auch kleine Plattformen an die schon erwähnte Hashdatenbanken anschließen. Die neue EU-Kommission plant zudem den sogenannten Digital Services Act, in dem die Verantwortlichkeit von Online-Plattformen bereichsübergreifend neu geregelt werden soll. Vieles ist derzeit noch offen. Das Worst-Case-Szenario wäre, dass das Internet aufgrund der Verantwortlichkeit der Plattformen am Ende ein großes Netflix würde, in dem nur Beiträge, die autorisiert sind, von bestimmten Teilnehmer*innen, denen die Plattform Vertrauen entgegenbringt, hochgeladen werden - also das Ende von User generated Content.

Müller-Helle: Würde diese Moderation oder Einhegung unter Umständen ins Gehege kommen mit dem Artikel 5 Absatz 3 des deutschen Grundgesetzes zur Kunstfreiheit? Frau Müller-Mall, könnte man sagen, dass die Rechtslage schwierig wird, wenn ich - ich konstruiere mal einen Fall - eine Internetkünstlerin bin, die terrorismusk ritische Kunst macht, welche auf Plattformen hochgeladen werden soll und sich bestimmter visueller Inhalte bedient, die in Hashdatenbanken zu finden sind. Diese kann ich dann nicht nutzen, weil sie eigentlich schon gekennzeichnet, identifiziert sind, was einen kritischen Umgang und auch eine künstlerische Reflektion in gewisser Weise einschränkt. Wäre dies ein Rechtsfall, weil der künstlerische Ausdruck eingeschränkt ist?

Müller-Mall: Es ist für mich nicht ganz einfach, dies für so einen Fall konkret einzuschätzen, weil ich die Kriterien dieses Zusammenschlusses nicht genau kenne und man kaum in allgemeiner Weise über ein Verbot künstlerischer Mittel sprechen kann, ohne eine Vorstellung von den konkreten künstlerischen Mitteln zu haben. Ganz grundsätzlich befindet man sich hier in einer fundamentalen Spannungslage. Wir wünschen uns einerseits eine Netzwelt, die so wenig wie möglich reguliert ist, und andererseits möchten wir natürlich auch die rechtlichen Strukturierungen und Wertungen, die wir in der analogen Welt vornehmen, nicht aufgeben. Ich sehe keinen Grund, warum diese in der digitalen Welt nicht gelten sollten. Die große Schwierigkeit besteht darin, in dieser Spannungslage sowohl normative als auch technische Lösungen zu finden, die auch noch nachvollziehbar sind. Das Problem der Opazität gilt ja für die demokratische Struktur selbst genauso wie für solche Entscheidungen und ihre technischen Bedingungen. Gleichzeitig sind wir auch aus ganz anderen Gründen weit entfernt von einem ,freien Netz', denn auch die digitale Welt ist durch Marktmechanismen und von kapitalistischen Logiken 
strukturiert. Ich würde den Einfluss dieser Logiken auf all diese Fragen in keiner Weise unterschätzen. Ich glaube auch, dass beispielsweise die Löschungspraxis oder auch die Kriterien, die Facebook anlegt, Konsequenzen aus diesen Marktmechanismen und ökonomischen Annahmen sind. Facebook will natürlich den durchschnittsamerikanischen Nutzer, die durchschnittsamerikanische Nutzerin nicht verprellen. Und daraus resultieren auch die Entscheidungen zur Löschung.

Müller-Helle: Ich würde gerne auf technikphilosophischer Ebene noch eine Sache ergänzen und zwar zur Opazität der Mechanismen, die uns jetzt schon das ganze Gespräch begleiten. Diese greift nämlich auch in der implementierten Technik selber. Bruno Latour hat das mit dem Begriff des Blackboxing beschrieben, eine technische Struktur, die sich in ihrem Funktionieren selbst unsichtbar macht. Das heißt, dass auf der technischen Ebene im reibungslosen Funktionieren von Löschungen oder Übertragungen von Bildern schon dieses Moment der Opazität steckt. Man kann in diese Prozesse nicht unmittelbar und nicht zu jedem Zeitpunkt eingreifen. Das sind dann auch Fragen, welche die Handlungsmacht und das Verstehen dieser technischen Vorgänge betreffen.

Was ich jetzt am Ende interessant finde, ist die Spannung aus der Bereitstellung eines großen Raumes der Entfaltung im Internet, der von Ihnen als Ermöglichung und Empowerment beschrieben wurde, und einer Notwendigkeit der Regulation. Die Wissenschaftshistorikerin Lorraine Daston hat einmal beschrieben, dass wir jetzt (wieder) in einer Schwellenphase sind, in der unsere gesamten Begriffe, Techniken und Repräsentationen noch einmal neu sortiert werden müssen, und dazu gehört, wie Sie beide ausgeführt haben, auch das Nachdenken über das juristische Urteilen angesichts der weit auseinander liegenden Pole von totaler Freiheit und normativer Regulierung im digitalen Zeitalter. Das Gespräch wurde am 24. August 2020 coronabedingt per ZOOM geführt und aufgezeichnet.

1 Zachary Small: Facebook Censors Art Historian for Posting Nude Art, Then Boots Him from Platform, 27.11.2018, Hyperallergic, https:/hyperallergic.com/472706/facebook-censors-art-historian-for-postingnude-art-then-boots-him-from-platform/ (Stand 9/2020).

2 Anm. der Redaktion: Facebook hat Ruben Cordova seiner Aussage nach nie kontaktiert, um die Umstände der Löschung seines Accounts aufzuklären. Cordova stellte jedoch fest, dass seine Posts ab dem 24. Januar 2019 von dem italienischen Kunsthistoriker Ivano Mazzini wieder getagged wurden, sein Account also wiederhergestellt worden war - einschließlich seines fotografischen Archivs. Zuvor hatte der Fall weitreichende mediale Aufmerksamkeit erlangt.

3 Vgl. hierzu Facebook's Community Standards, https://www.facebook.com/communitystandards/adult_ nudity_sexual_activity (Stand 27.9.2020).

4 Vgl. Digitale Gesellschaft (Hg.): Was sind Uploadfilter? Berlin 2020, S. 20.

5 Zum Christchurch-Attentat vgl. den Beitrag von Philipp Müller in diesem Band. 